\title{
Effects of calcium-dependent molecular chaperones and endoplasmic reticulum in the amygdala in rats under single-prolonged stress
}

\author{
BING XIAO, JIAN-GANG WANG, FANG HAN and YU-XIU SHI \\ Department of Histology and Embryology, Basic Medical Sciences College, China Medical University, \\ Shenyang, Liaoning 110122, P.R. China
}

Received May 21, 2017; Accepted October 17, 2017

DOI: $10.3892 / \mathrm{mmr} .2017 .7976$

\begin{abstract}
The purpose of the present study was to investigate the role of endoplasmic reticulum (ER)-resident molecular chaperone proteins to identify whether these proteins were involved in post-traumatic stress disorder (PTSD). The present study detected changes of calreticulin (CRT), calnexin (CNX) and ERp57 in the amygdala of rats, which may with aim of providing a novel insight into the modulation effect of amygdala in PTSD. Single-prolonged stress (SPS) was applied to create the models of PTSD in rats. The expression levels of CRT, CNX and ERp57 were examined using immunohistochemistry or immunofluorescence, western blot analysis and reverse transcription-quantitative polymerase chain reaction (RT-qPCR). The results showed that SPS induced significant changes in CRT, CNX and ERp57 expression levels. Furthermore, the expression levels of CRT, CNX and ERp57 were significantly upregulated when compared to that in the control group after SPS exposure by western blot analysis $(\mathrm{P}<0.05)$. RT-qPCR analysis supported these results, indicating an upregulation of mRNA expression level. Taken together, the present findings suggest that SPS may induce changes to the expression of CRT, CNX and ERp57 in the amygdala of rats. The present study provides an insight into the effects of ER-resident molecular chaperones in the amygdala participating in PTSD, and provides the experimental basis and a mechanism for the pathophysiology of PTSD.
\end{abstract}

\section{Introduction}

Post-traumatic stress disorder (PTSD) is a long-lasting mental disorder that develops after exposure to a traumatic event such as traffic collisions, sexual assault, natural disaster,

Correspondence to: Professor Yu-Xiu Shi, Department of Histology and Embryology, Basic Medical Sciences College, China Medical University, 77 Puhe Road, Shenyang, Liaoning 110122, P.R. China

E-mail: shiyuxiu223@sina.com

Key words: post-traumatic stress disorder, single prolonged stress, amygdala, calreticulin, calnexin. ERp57 or other threats. Symptoms that often last for more than a month or even years after the event may include continuous disturbing re-experience of the traumatic event, avoidance of trauma-related cues, hypervigilance and numbing of general responsiveness $(1,2)$. However, the pathological mechanisms of PTSD is not well clarified, although recent studies indicate that calcium ion disturbances, apoptosis, dysfunction of mitochondria or endoplasmic reticulum (ER) are involved in PTSD (3-6).

As a multifunctional organelle, the ER participated in multiple cellular functions, including production of glycogen and steroids, folding and transport of various proteins, sequestration of calcium and cell apoptosis (7-9). Physiological and pathological stimuli that disrupt ER homeostasis are responsible for dysfunction of ER or ER stress, including perturbation of calcium homeostasis, accumulative unfolded or misfolded proteins and viral infection. (10-12). Cells cope with ER stress via an adaptive unfolding protein response (UPR) $(13,14)$.

Both calreticulin (CRT) and calnexin (CNX) are ER resident calcium-binding chaperones (15-17) and play a vital role in the folding of newly synthesized proteins and quality control pathways in the ER $(16,18)$. ERp57 belongs to the protein disulfide isomerses family (PDIs), participating in the folding of newly synthesized glycoproteins, in collaboration with CRT and CNX (19-21). As folding proteins and chaperones, CRT, CNX and ERp57 also participate in dealing with misfolded proteins from the ER via its own unique mechanism (13). These folding proteins are important, since fluctuations of intraluminal $\mathrm{Ca}^{2+}$ level could affect ER function and induce cell death (22).

As part of the limbic system in brain, amygdala has long been considered as a pivotal brain structure responsible for anxiety, fear, learning and memory modulation associated with emotional events (23-25). Amygdala nuclei encompass several structures: Basolateral complex, cortical nucleus, medial nucleus, central nucleus, and the intercalated cell clusters (26). Among these nuclei subgroups, the basolateral nuclei play a major role in mediating anxiety, emotional arousal and memory (27), as indicated by many studies. Therefore, the present study focused on observing Single-prolonged stress (SPS)-induced changes of the basolateral nuclei.

Lower plasma cortisol level and enhanced inhibition of the hypothalamo-pituitary-adrenal (HPA) axis are 
neuroendocrinological mark of PTSD (28). SPS paradigms were shown to induce these changes and widely used in the studies of PTSD (29-31). In this study, we also created animal models by exposure to SPS. The purpose of this work was to investigate whether CRT, CNX and ERp57 were involved in dysfunction of amygdala on rats exposed to SPS using immunofluorescence, western blot and qPCR to assess the changes, which might provide novel insights into the pathogenesis of PTSD and experimental basis for new treatments.

\section{Materials and methods}

Animals. Eighty male Wistar rats weighing 150-160 g at the start of the study, were supplied by Changsheng Biotechnology Co., Ltd. (Liaoning, China). Rats were housed 2-3 animals per plastic cage on a $12 \mathrm{~h}$ light-dark schedule at $22 \pm 2^{\circ} \mathrm{C}$ with free access to water and food for 7 days. All procedures were conducted in conformity with Guidance for the Care and Use of Laboratory Animals, the National Institute of Health. The study was approved by the Ethics Committee of Laboratory Animal Welfare and Ethics (China Medical University) All efforts were made to reduce the number of animals used and to minimize animal suffering during the experiment.

Grouping and model establishment. Animals were divided randomly into four groups: 1) the control group (Cont); 2) SPS 1 day group; 3) SPS 7 days group; 4) SPS 14 days group. Control animals remained in their home cages with no handling for 7 or 14 days and were sacrificed at the same time as the SPS groups. The SPS-rats underwent the SPS procedure on the first day and remained in their cages until sacrifice. The SPS protocol was based on a combined plural stress paradigm $(29,30)$ : Immobilization (compression with plastic bags) for $2 \mathrm{~h}$, followed by forced swimming for $20 \mathrm{~min}$ in a plexiglass cylinder $\left(40 \mathrm{~cm}\right.$ depth; $23 \pm 2^{\circ} \mathrm{C}$ ), and then rest for $15 \mathrm{~min}$, ether anesthesia until loss of consciousness at last. Cervical dislocation was used as the method of sacrifice.

Measurement of animal body weight. The body weights of both the control and SPS groups' rats were recorded every other day, and then the body weight growth curve was drawn based on the average weight in each group of rats.

Perfusion based sections. Rats of each group were perfused via left ventricle and fixed with $250 \mathrm{ml}$ of pre-cold heparinized $0.9 \%$ saline, followed by $300 \mathrm{ml}$ of $4 \%$ paraformaldehyde in $0.01 \mathrm{M}$ phosphate buffer ( $\mathrm{pH} 7.2)$. Then the brain tissues were rapidly separated and fixed in $4 \%$ PFA for $6 \mathrm{~h}$ at $4^{\circ} \mathrm{C}$, and were immersed in a $30 \%$ sucrose solution in $0.01 \mathrm{M}$ phosphate-buffered saline (PBS; pH 7.2) at $4^{\circ} \mathrm{C}$ after then. Tissues were fast frozen in liquid nitrogen and sectioned coronally. Frozen sections $(12 \mu \mathrm{m})$ were prepared for immunohistochemistry or immunofluorescence analysis using a cryostat (CM 3050; Leica, Mannheim, Germany).

Immunofluorescence analysis of CRT. After being washed with 0.01 M PBS for three times, the sections of each group were incubated with $5 \%$ bovine serum albumin (BSA) for $30 \mathrm{~min}$ to block non-specific staining at room temperature (RT). The sections were then incubated with mouse monoclonal
anti-CRT antibody (1:200; Santa Cruz Biotechnology, Inc., Santa Cruz, CA, USA) in $0.01 \mathrm{M} \mathrm{PBS}$ overnight at $4^{\circ} \mathrm{C}$. After being washed with PBS for three times, the sections were incubated with Cy3-conjugated goat anti-mouse IgG (1:50; Boster, Wuhan, China) for $2 \mathrm{~h}$ at RT. After being washed with PBS, slices were then mounted with glycerin and observated by fluorescence microscope.

Five slides were randomly selected from each group and five visual fields in the basolateral amygdala were randomly selected from each slide (magnification, x400). We recorded the fluorescent intensity of CRT-immunopositive cells to evaluate the average fluorescent intensity using a Meta Morph/DPIO/BX41 morphology image analysis system.

Immunohistochemical analysis of CNX and ERp57. After being washed with PBS three times, the sections of each group were incubated with 5\% BSA for $30 \mathrm{~min}$ to block non-specific staining at RT. The sections were then incubated with goat polyclonal anti-CNX antibody (1:200; Santa Cruz Biotechnology, Inc.) or mouse monoclonal anti-ERp57 antibody (1:200; Santa Cruz, USA) overnight at $4^{\circ} \mathrm{C}$. The sections were incubated with rabbit anti-goat $\operatorname{IgG}(1: 50$; Boster) or goat anti-mouse $\operatorname{IgG}\left(1: 50\right.$; Boster) for $2 \mathrm{~h}$ at $37^{\circ} \mathrm{C}$ and then with streptomycin-avidin-biotin-peroxidase complex (SABC) for $20 \mathrm{~min}$ at $37^{\circ} \mathrm{C}$. The sections were washed three times with PBS after each of incubation and subsequently incubated with 3,3'-diaminobenzidine (DAB).

Five slides were randomly selected from each group and five visual fields in the basolateral amygdala were randomly selected from each slide (magnification, x400). We recorded the optical density (OD) of positive cells in each field to evaluate the average OD value. The OD of immunoreactivity of CNX or ERp57-immunopositive cells were analyzed using a MetaMorph/DPIO/BX41 morphology image analysis system.

Western blot analysis to detect CRT, CNX and ERp57. Rats of each group were decapitated, and the brains were removed rapidly and the basolateral amygdala was separated immediately on ice. After being washed twice with cold $0.01 \mathrm{M}$ PBS, the tissues were homogenized with RIPA Lysis buffer respectively. The supernatant liquor was collected, and then concentration of protein was measured respectively via BCA kit. Equal amounts of protein $(50 \mu \mathrm{g} /$ lane $)$ prepared from each tissue was separated by $10 \%$ (w/v) SDS-PAGE (110 V) and transferred onto a PVDF membrane via electroblotting for $70 \mathrm{~min}$ at $350 \mathrm{~mA}$. After being blocked with $5 \%$ dried skim milk in $0.05 \%$ Tween-20-TBST at RT for $3 \mathrm{~h}$, the membrane was incubated with mouse anti-CRT (1:500), goat anti-CNX (1:500) or mouse anti-ERp57 (1:200; all Santa Cruz Biotechnology, Inc.) overnight at $4^{\circ} \mathrm{C}$.

The membrane were washed three times with 0.01 M TBST and incubated with the HRP-conjugated secondary antibody for $2 \mathrm{~h}$ at RT. Then the blots were visualized by enhanced chemiluminescence (ECL; Beyotime Biotechnology, Jiangsu, China). To confirm equal protein loading the same blots were reincubated with antibodies against GAPDH (1:1,000; Boster). Immunoreaction for GAPDH was also detected by the ECL. The OD were analyzed on the Gel Image Analysis System. The levels of proteins were evaluated by calculating the OD ratio of CRT/GAPDH, CNX/GAPDH and ERp57/GAPDH. 


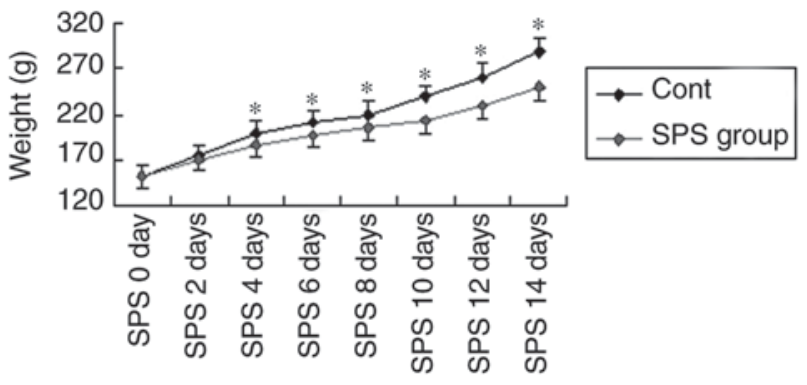

Figure 1. The body weight growth curve of the control and SPS group. Rats in the normal control group showed natural growth and the body weight increased relatively quickly. The results indicated slower increase in the SPS groups compared with those in the normal control group and statistically significant ( $\mathrm{P}<0.05$ vs. the Cont group). SPS, single-prolonged stress.
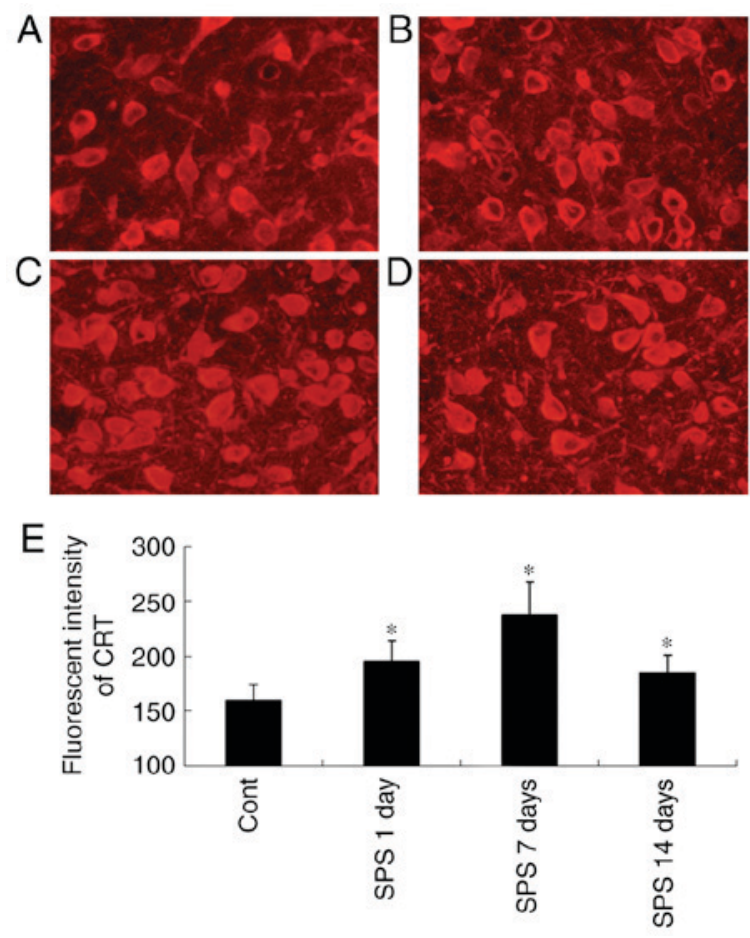

Figure 2. Presentation of CRT expression in the basolateral amygdala in the each group (A-D; magnification, $\mathrm{x} 400$ ). A, Cont group; B, SPS 1 day group; C, SPS 7 days group; D, SPS 7 days group) Quantitative analysis results of fluorescent intensity (E) ${ }^{*} \mathrm{P}<0.05$ vs. the Cont group. CRT, calreticulin; SPS, single-prolonged stress.

Quantitative real-time reverse transcription-PCR to detect $C R T, C N X$ and ERp57. Total mRNA from the basolateral amygdala of each group was extracted according to the protocol of Trizol (Takara Biotech, Otsu, Japan) and $1 \mu \mathrm{g}$ of total RNA was reverse transcribed into cDNA. Then the cDNA was used as a template in RT-PCR amplifications performed via a SYBR Real-Time PCR kit (Takara Biotech, Dalian, China). The following primers were used: CRT (upper, 5'-TTCTTGGACGGAGATGCCTG-3' and lower, 5'-GGT CCCCGTAGAATTTGCCA-3'), CNX (upper, 5'-CCGGGA GGCTCGAGATAGA-3' and lower, 5'-ATCCACCCTGAC AGAGACCC-3'), GAPDH (upper, 5'-GGCACAGTCAAG GCTGAGAATG-3', and lower, 5'-ATGGTGGTGAAGACG CCAGTA-3'). All primers were synthetized by Shenggong
Biotech Company (Shanghai, China) according to the serial number from Genbank. The results were analyzed using the Rotor Genne PCR-3000 (Corbett Research, Sydney, Australia). Relative mRNA levels were calculated using the $2^{-\Delta \Delta \mathrm{Ct}}$ method and normalized against.

Statistical analysis. All the experiment results were analyzed by one-way analysis of variance (ANOVA) using SPSS 23.0 software. All data were expressed as means \pm standard error. $\mathrm{P}<0.05$ was considered to indicate a statistically significant difference.

\section{Results}

Decreased animal body weight after SPS stimuli. Compared with the normal control, rats after SPS stimuli presented loss of appetite. Accordingly, the body weight growth curve reflected this difference. As shown in Fig. 1, rats in the control group showed a normal increase in body weight over time, rats in the model group presented lighter weight after stimulation $(\mathrm{P}<0.05)$.

Immunofluorescence staining analysis results of CRT. CRT-ir was were shown in Fig. 2. via immunofluorescence staining. The CRT-ir was located in cytoplasm (Fig. 2A-D). In the Cont group, weak fluorescent intensity of CRT-positive cells was shown in Fig. 2A, and that of SPS rats were significantly strong compare to the Cont group $(\mathrm{P}<0.01)$ (Fig. 2E).

Immunohistochemical staining analysis results of CNX and ERp57. As was shown in Fig. 3, CNX and ERp57 widely distributed in the cytoplasm, and also around the nucleus of cells in immunohistochemical staining. Evaluation of CNX and ERp57 by immunohistochemical indicated a stronger positive immunoreaction in the SPS model groups compared with the Cont group. As shown in Fig. 3I, the histogram indicated this change.

Western bloting analysis protein expression levels. Molecular weights of CRT, CNX and ERp 57 were 64, 90, and $57 \mathrm{kDa}$, respectively, showing clear bands detected by western blot. Evaluated by calculating the OD ratio of CRT/GAPDH, CNX/GAPDH and ERp57/GAPDH, the level of protein expression indicated a marked upregulation after SPS stimuli and peaked at SPS 7 days group compared with that of the Cont group $(\mathrm{P}<0.05)$ (Fig. 4).

Quantitative real-time PCR analysis results of CRT, CNX and ERp57. Certain expression of CRT, CNX and ERp 57 mRNA presented in amygdala neurons of normal control rats (Fig. 5). The levels of CRT, CNX and ERp 57 mRNA were normalized with GAPDH mRNA. The expression of CRT, CNX and ERp 57- mRNA appeared an obvious increase after exposure to SPS and began decline on SPS 14-day $(\mathrm{P}<0.05)$ (Fig. 5).

\section{Discussion}

PTSD is an emotional illness and has long been thought to involve a dysfunction in reaction to fear-related stimulation. Many 


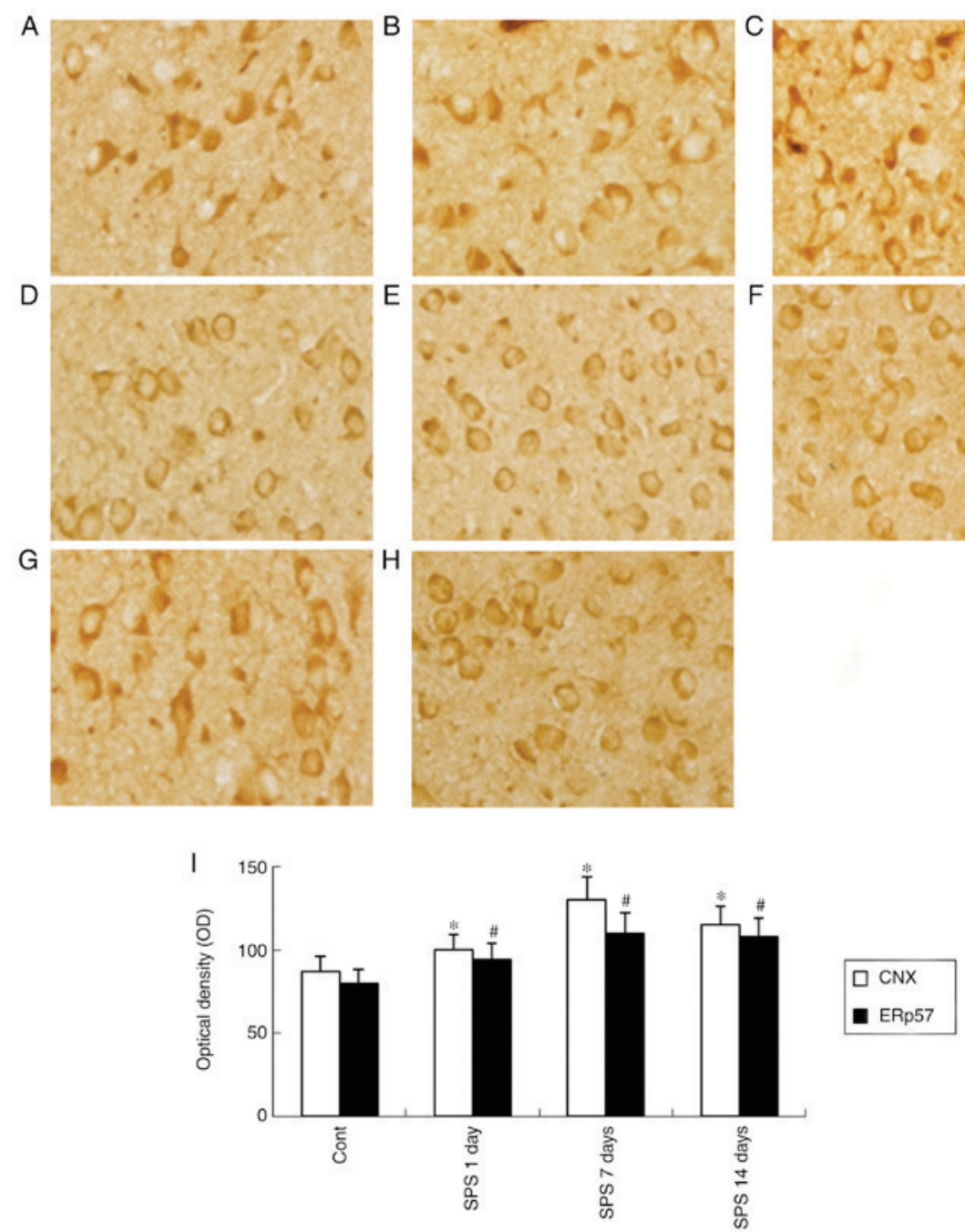

Figure 3. Presentation of CNX and ERp57 expression in the basolateral amygdala in each group. (A-C and G) CNX; (D-F and H) ERp57 (magnification, $\mathrm{x} 400$ ). (A and D) Cont group; (B and E) SPS 1 day group; (C and F) SPS 7 days group; (G and H) SPS 7 days group semi-quantitative analysis results of optical density (I) ${ }^{*} \mathrm{P}<0.05$ vs. the Cont group, ${ }^{*} \mathrm{P}<0.05$ vs. the Cont group. CNX, calnexin; SPS, single-prolonged stress.

A

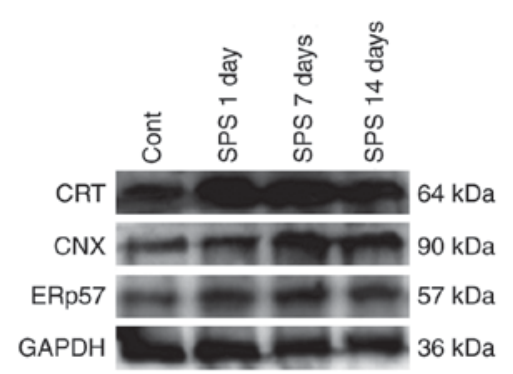

B

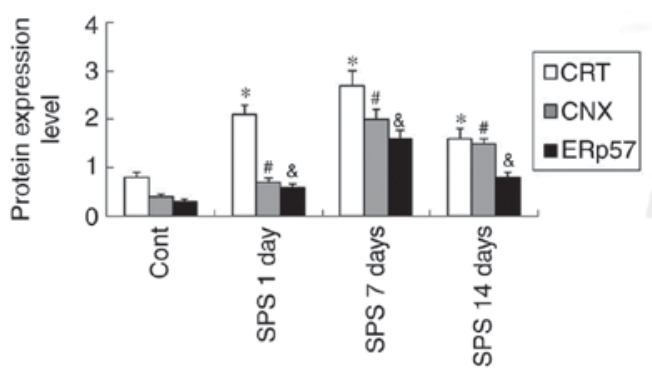

Figure 4. Western blot analysis of CRT, CNX and ERp57 (A). Quantitative analysis results of fluorescent intensity of optical density (B). ${ }^{*} \mathrm{P}<0.05$ vs. control group, ${ }^{\#} \mathrm{P}<0.05$ vs. the Cont group, ${ }^{\&} \mathrm{P}<0.05$ vs. the Cont group. CRT, calreticulin; CNX, calnexin; ER, endoplasmic reticulum; SPS, single-prolonged stress.

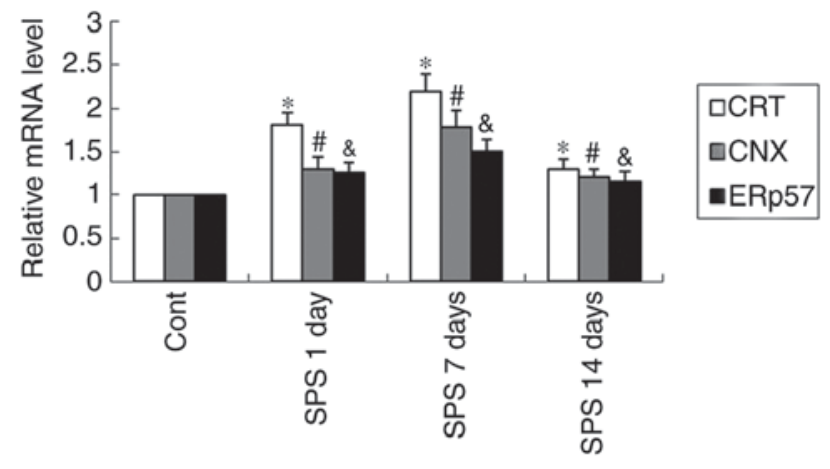

Figure 5. Quantitative real-time PCR Analysis Results of CRT, CNX and ERp57. ${ }^{\mathrm{P}}<0.05$ vs. the Cont group, ${ }^{\prime} \mathrm{P}<0.05$ vs. the Cont group, ${ }^{\text {\& }} \mathrm{P}<0.05$ vs. the Cont group. CRT, calreticulin; CNX, calnexin; ER, endoplasmic reticulum; SPS, single-prolonged stress.

lines of evidence confirmed the crucial role of amygdala in the processing of fear-related expression based on investigations on animals and humans $(24,25)$, and multiple studies have indicated the basolateral amygdalaas a key area for regulating stress-related memory $(27,32)$. 
As a second messenger molecule in the cell, calcium ion plays an important role in development and participating in many cellular processes. The majority of intracellular calcium ion is stored in the lumen of the ER. Calcium ion homeostasis play a vital role in functionating of ER, and disturbance of calcium homeostasis caused by various factors disrupt correct protein folding, which could induce an accumulation of misfolded proteins, or ER stress (13). Our previous work has indicated that SPS induces $\mathrm{Ca}^{2+}$ overload in the amygdala neurons of rats after SPS stimuli (33). In this study, we detected changes of $\mathrm{Ca}^{2+}$ buffering protein. As the master regulator of protein quality-control system and molecular chaperones in the ER, CRT and CNX appeared significant upregulation in the amygdala neurons after SPS stimuli, and peaked at SPS 7 days. It appears resonable to suppose that the changes of CRT and CNX are compensatory up-regulation to provide cytoprotection in response to $\mathrm{Ca}^{2+}$ overload. ERp57 participates in the folding of newly synthesized glycoproteins and dealing with misfolded proteins from the ER via its own unique mechanism, in concert with CRT and CNX (13,19-21). We also detected changes of ERp57 in the amygdala neurons in this study. Similarly, the results showed that ERp57 upregulated significantly after SPS stimulation. It appears resonable to suppose that all these changes are compensatory in order to alleviate cell damage, however, this compensatory capacity is limited. Then downregulation of these proteins appeared at SPS 14 days when it beyond its own compensatory capacity.

In this study, we investigated changes of CRT, CNX and ERp57 in the amygdala of rats to find these ER-resident molecular chaperone whether or not participate in PTSD, using immunofluorescence, western blot and real-time PCR to measure the protein and mRNA levels. Taken together, we found CRT, CNX and ERp57 upregulated significantly in the amygdala of rats after exposure to SPS. The results of qPCR are consistent with western blot. It appears resonable to suppose that that $\mathrm{Ca}^{2+}$ overload after SPS stimuli induced accumulation of misfolded protein, which lead to upregulation of CRT, CNX and ERp57 in order to deal with accumulation of misfolded protein by folding once again to ease cell damage. Nevertheless, excessive misfolded or unfolded proteins resulted in dysfunction of ER in amygdala neurons, which might be involved in pathogenesis for abnormality of affect and behavior induced by PTSD. This findings provide new insight into the pathogenesis of PTSD. However, it remains unclear as to whether the changes of chaperones proteins serve as a trigger or a consequnce of amygdala neuron dysfunction now.

Up till now, the pathological mechanisms of PTSD are not yet understood in spite of extensive investigations. PTSD may induce series of biological and functional abnormalities of the amygdala and other brain regions, which results in dysfuction of brain finally. The present study shed some light on the effects of ER-resident molecular chaperone participating in PTSD, which might provide experimental basis and a mechanism for the pathophysiology of PTSD. Further studies on the regulatary mechanisms of molecular chaperone on neuronal function in PTSD also should be included. So, there is a need for more in-depth research on PTSD.

\section{Acknowledgements}

The present study was funded by a grant from the National Natural Science Foundation of China (no. 31200772) and Shenyang Science and Technology Project (no. F16-2-5-1-35).

\section{References}

1. American Psychiatric Association: Diagnostic and Statistical Manual of Mental Disorders. 5th edition. Arlington, VA, American Psychiatric Publishing, pp271-280, 2013.

2. Mol SS, Arntz A, Metsemakers JF, Dinant GJ, Vilters-van Monfort PA and Knottnerus JA: Symptoms of post-traumatic stress disorder after non-traumatic events: Evidence from an open population study. Br J Psychiatry 186: 494-499, 2005.

3. Wen Y, Li B, Han F, Wang E and Shi Y: Dysfunction of calcium/calmodulin/CaM kinase II $\alpha$ cascades in the medial prefrontal cortex in post-traumatic stress disorder. Mol Med Rep 6: 1140-1144, 2012.

4. Zhao D, Han F and Shi Y: Effect of glucose-regulated protein 94 and endoplasmic reticulum modulator caspase-12 in medial prefrontal cortex in a rat model of posttraumatic stress disorder. J Mol Neurosci 54: 147-155, 2014.

5. Xie J, Han F and Shi Y: The unfolded protein response is triggered in rat neurons of the dorsal raphe nucleus after single-pronged stress. Neurochem Res 39: 741-747, 2014.

6. Zhao W, Han F and Shi YX: IRE1 $\alpha$ pathway of endoplasmic reticulum stress induces neuronal apoptosis in the locus coeruleus of rats under single prolonged stress. Prog Neuropsychopharmacol Biol Psychiatry 69: 11-18, 2016.

7. Groenendyk J and Michalak M: Endoplasmic reticulum quality control and apoptosis. Acta Biochim Pol 52: 381-395, 2005.

8. Breckenridge DG, Germain M, Mathai JP, Nguyen M and Shore GC: Regulation of apoptosis by endoplasmic reticulum pathways. Oncogene 22: 8608-8618, 2003.

9. Xiang J, Gu X, Qian S and Chen Z: Endoplasmic reticulum stress-mediated apoptosis involved in indirect recognition pathway blockade induces long-term heart allograft survival. J Biomed Biotechnol 2010: 705431, 2010.

10. Lv S, Sun EC, Xu QY, Zhang JK and Wu DL: Endoplasmic reticulum stress-mediated autophagy contributes to bluetongue virus infection via the PERK-eIF2 $\alpha$ pathway. Biochem Biophys Res Commun 466: 406-412, 2015.

11. Rao RV, Hermel E, Castro-Obregon S, del Rio G, Ellerby LM, Ellerby HM and Bredesen DE: Coupling endoplasmic reticulum stress to the cell death program. Mechanism of caspase activation. J Biol Chem 276: 33869-33874, 2001.

12. Boyce M and Yuan J: Cellular response to endoplasmic reticulum stress: A matter of life or death. Cell Death Differ 13: 363-373, 2006.

13. Prell T, Lautenschläger J and Grosskreutz J: Calcium-dependent protein folding in amyotrophic lateral sclerosis. Cell Calcium 54: 132-143, 2013.

14. Bernales S, Papa FR and Walter P: Intracellular signaling by the unfolded protein response. Annu Rev Cell Dev Biol 22: 487-508, 2006.

15. Jung $\mathrm{J}$ and Michalak M: Cell surface targeting of myelin oligodendrocyte glycoprotein (MOG) in the absence of endoplasmic reticulum molecular chaperones. Biochim Biophys Acta 1813: 1105-1110, 2011.

16. Williams DB: Beyond lectins: The calnexin/calreticulin chaperone system of the endoplasmic reticulum. J Cell Sci 119: 615-623, 2006

17. Hebert DN and Molinari M: In and out of the ER: Protein folding, quality control, degradation, and related human diseases. Physiol Rev 87: 1377-1408, 2007.

18. Itakura M, Tsujimura J, Yamamori S, Ohkido T and Takahashi M: NMDA receptor-dependent recruitment of calnexin to the neuronal plasma membrane. Neurosci Lett 550: 173-178, 2013.

19. Ellgaard L, Riek R, Herrmann T, Güntert P, Braun D, Helenius A and Wüthrich K: NMR structure of the calreticulin P-domain. Proc Natl Acad Sci USA 98: 3133-3138, 2001.

20. Coe $\mathrm{H}$ and Michalak M: ERp57, a multifunctional endoplasmic reticulum resident oxidoreductase. Int J Biochem Cell Biol 42: 796-799, 2010. 
21. Ellgaard L and Frickel EM: Calnexin, calreticulin, and ERp57: Teammates in glycoprotein folding. Cell Biochem Biophys 39: 223-247, 2003.

22. Szydlowska K and Tymianski M: Calcium, ischemia and excitotoxicity. Cell Calcium 47: 122-129, 2010.

23. Amunts K, Kedo O, Kindler M, Pieperhoff P, Mohlberg H, Shah NJ, Habel U, Schneider F and Zilles K: Cytoarchitectonic mapping of the human amygdala, hippocampal region and entorhinal cortex: Intersubject variability and probability maps. Anat Embryol (Berl) 210: 343-352, 2005.

24. Davis M and Whalen PJ: The amygdala: Vigilance and emotion. Mol Psychiatry 6: 13-34, 2001

25. Pape HC: Petrified or aroused with fear: The central amygdala takes the lead. Neuron 67: 527-529, 2010.

26. Solano-Castiella E, Anwander A, Lohmann G, Weiss M, Docherty C, Geyer S, Reimer E, Friederici AD and Turner R: Diffusion tensor imaging segments the human amygdala in vivo. Neuroimage 49: 2958-2965, 2010.

27. Young MB and Thomas SA: M1-muscarinic receptors promote fear memory consolidation via phospholipase $\mathrm{C}$ and the M-current. J Neurosci 34: 1570-1578, 2014.

28. Mihaljević S, Vuksan-Ćusa B, Marčinko D, Koić E, Kušević Z and Jakovljević M: Spiritual well-being, cortisol, and suicidality in croatian war veterans suffering from PTSD. J Relig Health 50: 464-473, 2011.
29. Yamamoto S, Morinobu S, Takei S, Fuchikami M, Matsuki A, Yamawaki S and Liberzon I: Single prolonged stress: Toward an animal model of posttraumatic stress disorder. Depress Anxiety 26: 1110-1117, 2009 .

30. Wang W, Liu Y, Zheng H, Wang HN, Jin X, Chen YC, Zheng LN, Luo XX and Tan QR: A modified single-prolonged stress model for post-traumatic stress disorder. Neurosci Lett 441: 237-241, 2008.

31. Khan S and Liberzon I: Topiramate attenuated exaggerated acoustic startle in an animal model of PTSD. Psychopharmacology (Berl) 172: 225-229, 2004.

32. Chavez CM, McGaugh JL and Weinberger NM: The basolateral amygdala modulates specific sensory memory representations in the cerebral cortex. Neurobiol Learn Mem 91: 382-392, 2009.

33. Xiao B, Han F and Shi YX: Dysfunction of Ca2+/CaM kinase IIalpha cascades in the amygdala in post-traumatic stress disorder. Int J Mol Med 24: 795-799, 2009. 